\title{
The Effects of Light Pollution on Amateur Astronomy
}

\author{
David Crawford
}

Kitt Peak National Observatory, P.O. Box 26732, Tucson, AZ 85726, U.S.A.

\begin{abstract}
The rapid increase in urban lighting, with the resultant increase in sky glow due to this lighting, has already severely affected both professional and amateur astronomy. It is not possible to observe as faint as was possible in the past from sites anywhere near large cities. Even those sites near smaller cities are compromised.

There are other adverse effects of outdoor lighting. One in particular affects amateur astronomers (more than professionals, in fact). That is light trespass from a (usually) nearby source shining directly into the amateur's observatory. It ruins dark adaption and can even affect photographic or photoelectric observations.

Astronomers are pushing for communities and individuals to apply "solutions", which can help greatly. These are: use the correct amount of light for the lighting task, not overkill; use controls on the lighting, such as shielding and correct placement (direct the light downward), and time controls; use low pressure sodium light sources whenever possible (especially in the vicinity of professional observing sites).

Without the widespread application of such solutions, amateurs, professionals, and even the general public will likely lose forever their superb view of the universe, as seen from a prime dark sky site. These solutions also maximize the quality of any lighting installation, often at lower cost than bad lighting.
\end{abstract}

